672

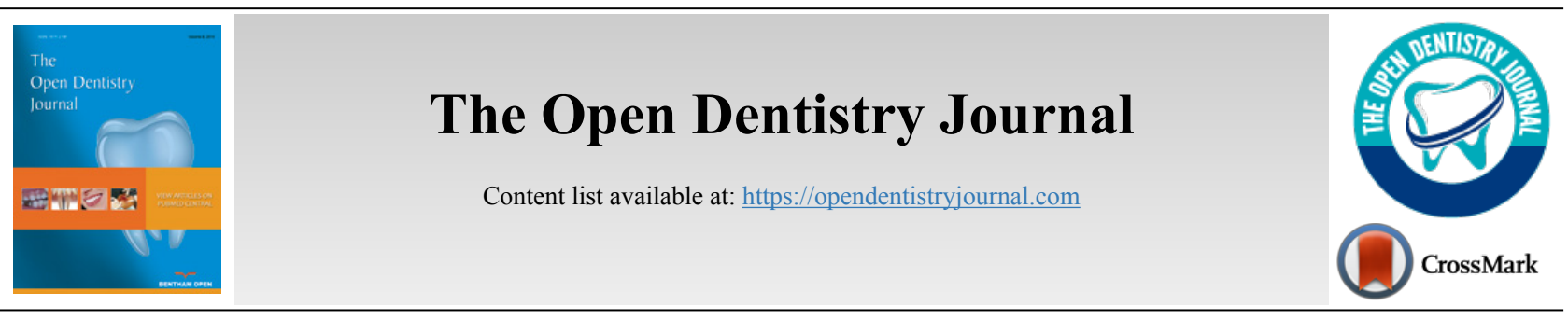

RESEARCH ARTICLE

\title{
Is Dental Panoramic Radiography Reliable to Assess Position of Bicortical Fixation Screws in Mandibular Fractures? Case Report
}

\author{
Ahmad Albassal $^{1}$, Nuraldeen Maher Al-Khanati ${ }^{1,2, *}$ and Munir Harfouch ${ }^{1}$ \\ ${ }^{\prime}$ Department of Oral and Maxillofacial Surgery, Faculty of Dental Medicine, Damascus University, Damascus, Syria \\ ${ }^{2}$ Department of Oral and Maxillofacial Surgery, Faculty of Dentistry, Syrian Private University, Damascus, Syria
}

\begin{abstract}
:
Background:

Panoramic radiography is widely used as a diagnostic tool before oral surgeries and can be considered the foremost follow-up image after. It provides a broad observation of the maxillomandibular complex at a lower cost and radiation dose. But cone-beam computed tomography (CBCT) examination, after panoramic radiograph evaluation, can produce a change in the diagnostic thinking of maxillofacial surgeons leading to alterations in treatment plans.

Objective:

We aim to report a case with incidentally discovered radiographic findings where diagnostic changes were caused by switching from panoramic to CBCT imaging.

Conclusion:

Radiographic assessment of the position and angulation of screws by panoramic x-ray should be approached with extreme caution. The image of choice is CBCT if nerve injury is suspected.
\end{abstract}

Keywords: Mandibular fracture, Nerve injury, Paresthesia, Panoramic radiography, CBCT, Bicortical fixation screws.

\begin{tabular}{|l|c|c|c}
\hline Article History & Received: May 21, 2021 & Revised: October 11, 2021 & Accepted: November 27, 2021
\end{tabular}

\section{DEAR EDITOR,}

Cone-beam computed tomography (CBCT) has been declared a new trend in the dental imaging practice and has been commonly used in almost every dental specialty, including oral and maxillofacial surgery [1]. It resolves the most critical drawback of two-dimensional (2D) images by presenting high-resolution multi-planar superimposition-free images of the jaws and facial structures. However, panoramic radiography is widely used as a diagnostic tool while developing treatment plans before oral surgeries and is still considered the foremost follow-up image. It provides a broad, single-film observation of the maxillomandibular complex at a lower cost and lower radiation dose. CBCT examination, following panoramic radiograph evaluation, can produce change in the diagnostic thinking of oral and maxillofacial surgeons leading to alterations in treatment plans of impacted

\footnotetext{
* Address correspondence to this author at the Department of Oral and Maxillofacial Surgery, Faculty of Dentistry, Syrian Private University, P.O. Box 36822, Damascus, Syria; Tel: +963 956589272;

E-mail: nuraldeen.alkhanati@gmail.com
}

third molars [2]. This could be applicable also in regard to diagnosis and follow-up of other oral and maxillofacial surgeries, e.g. mandibular fracture management. There are, to the best of our knowledge, no prior publications indicating the validity of panoramic radiography in assessing the position of open reduction and internal fixation (ORIF)'s screws and their proximity to vital structures, i.e. inferior alveolar nerve and vessels. Hereby, we aim to report this case with incidentally discovered radiographic findings where diagnostic changes were caused by CBCT.

A 26-year-old male was referred to Oral and Maxillofacial Surgery Department (Faculty of Dental Medicine, Damascus University) with a history of mandibular right para-symphysis fracture and surgical management with ORIF four months ago. He complained of numbness of the right side of his lower lip. Blood-based tests showed negative results regarding potential titanium allergy. We explained to the patient that this is a possible complication due to potential inferior alveolar nerve injury after mandibular fractures. But on radiographic 
evaluation, we thought that the culprit for the persistence of labial paresthesia until that time could be the titanium bicortical screw used for ORIF which was apparently oriented to touch the inferior border of the mandibular canal on panoramic radiography (Fig. 1a). The patient was told then that this issue might be irreversible and he had to get used to the current situation. Later on, the same patient returned to us seeking surgical management of his impacted third molars. CBCT of the jaws was performed to assess the position of his mandibular third molars in relation to the mandibular canal and to evaluate the difficulty of surgical extractions in order to take the most appropriate management. It was gladdening and surprising that the patient reported a huge positive improvement in lower lip sensations in the meantime of two months from his last visit. Reassessment of the screws was done via the patient's CBCT (Fig. 1b). The CBCT was viewed and the mandibular canal was traced using Ez3D Plus V1.2 software (Vatech Co. Ltd., Gyeonggi-do, Korea). By comparing both patient's radiographs (Fig. 1), it was very clear that the screws' orientation in different directions, as appeared on the panorama, was not true. The CBCT confirmed that these bicortical screws were parallel to each other and acceptably oriented in relation to the cortical plates of mandible and mandibular canal. This made us step back and admit that the panoramic radiograph misled us.

A

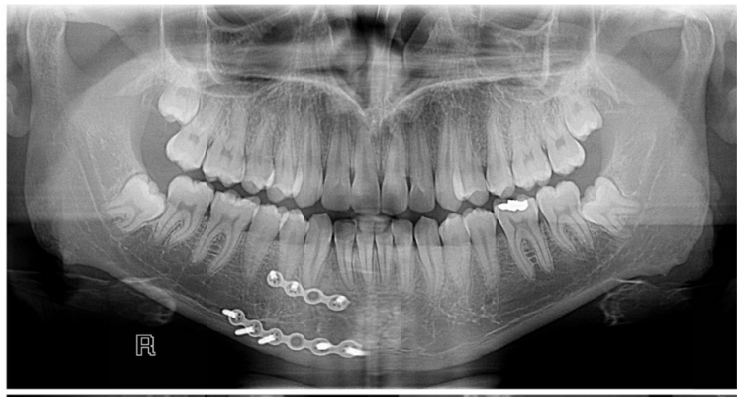

B

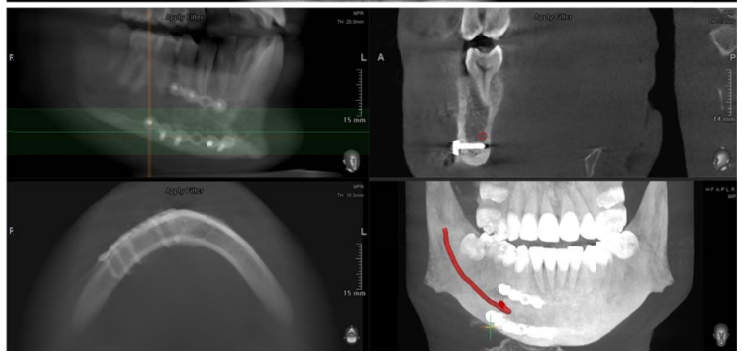

Fig. (1). Radiographs of the patient: (A) The panoramic X-ray showing two bone plates and screws used for mandibular fracture fixation. The distally-oriented first right screw in touch with mandibular canal and rest of screws oriented in different directions. (B) Cone-beam computed tomography (CBCT) displaying the case in different planes and $3 \mathrm{D}$ view with traced inferior alveolar nerve.

By reviewing the literature, we found that a similar finding was shown by Abbassy et al. [3]. They evaluated mini-implant sites in the posterior maxilla using traditional radiographs and CBCT [2]. Traditional 2D radiographs (i.e. panoramic and periapical) were not accurate enough, in comparison to $\mathrm{CBCT}$, in detecting the precise positions of mini-implants [3].
Watanabe et al. also found that it was highly difficult to diagnose root contact with orthodontic mini-implants using 2D radiography [4]. Although digital panoramic dental radiographs are known to be better than conventional panoramic radiography as diagnostic images, they may cause a misdiagnosis in some cases [5]. The extent of distortion in these images is influenced by the target object size and position in the focal trough [6]. The dental arch and the holding jaw reveal an increased curvature at some points, and panoramic images thus display greater distortion there [7]. More variations can be seen in the horizontal than vertical direction [6].

\section{CONCLUSION}

In conclusion, the radiographic assessment of the position and angulation of screws by panoramic radiography should be approached with extreme caution with an understanding of the inherent image magnification and distortion, especially in the horizontal direction. Selection between panoramic and CBCT follow-up images should be on a case-by-case basis. The image of choice is CBCT, if nerve injury is suspected. Further studies are warranted to evaluate the extent of distortion in bicortical screws' position and direction on the panoramic x-ray.

\section{CONFLICT OF INTEREST}

The authors declare no conflict of interest, financial or otherwise.

\section{ACKNOWLEDGEMENTS}

Declared none.

\section{REFERENCES}

[1] Burgess J. Digital DICOM in Dentistry. Open Dent J 2015; 9: 330-6. [http://dx.doi.org/10.2174/1874210601509010330] [PMID: 26464603]

[2] Mendonça LM, Gaêta-Araujo H, Cruvinel PB, et al. Can diagnostic changes caused by cone beam computed tomography alter the clinical decision in impacted lower third molar treatment plan?. Dentomaxillofac Radiol 2021; 50(4): 20200412.

[http://dx.doi.org/10.1259/dmfr.20200412] [PMID: 33197216]

[3] Abbassy MA, Sabban HM, Hassan AH, Zawawi KH. Evaluation of mini-implant sites in the posterior maxilla using traditional radiographs and cone-beam computed tomography. Saudi Med J 2015; 36(11): 1336-41.

[http://dx.doi.org/10.15537/smj.2015.11.12462] [PMID: 26593168]

[4] Watanabe H, Deguchi T, Hasegawa M, Ito M, Kim S, TakanoYamamoto T. Orthodontic miniscrew failure rate and root proximity, insertion angle, bone contact length, and bone density. Orthod Craniofac Res 2013; 16(1): 44-55.

[http://dx.doi.org/10.1111/ocr.12003] [PMID: 23311659]

[5] Albassal A, Al-Khanati NM, Harfouch M. Could a digital panoramic $\mathrm{X}$-ray not detect a displaced fracture of the mandible? Quant Imaging Med Surg 2021; 11(8): 3890-2.

[http://dx.doi.org/10.21037/qims-20-1053] [PMID: 34341757]

[6] Devlin H, Yuan J. Object position and image magnification in dental panoramic radiography: A theoretical analysis. Dentomaxillofac Radiol 2013; 42(1): 29951683.

[http://dx.doi.org/10.1259/dmfr/29951683] [PMID: 22933529]

[7] Tepedino M, Cornelis MA, Chimenti C, Cattaneo PM. Correlation between tooth size-arch length discrepancy and interradicular distances measured on $\mathrm{CBCT}$ and panoramic radiograph: An evaluation for miniscrew insertion. Dental Press J Orthod 2018; 23(5): 39.e1-39.e13.

[http://dx.doi.org/10.1590/2177-6709.23.5.39.e1-13.onl] [PMID: 30427499] 